\title{
A Variance based Approach (VBA) Digital Watermarking in Frequency Domain and Comparative Analysis using Walsh and Hadamard transform
}

\author{
H. M. Khalid Raihan Bhuyan \\ Assam Don Bosco University \\ Assam \\ India
}

\author{
Mriganka Gogoi \\ Assam Don Bosco University \\ Assam \\ India
}

\begin{abstract}
The paper introduces a Digital Image Watermarking technique based on Transform domain. The purpose of doing the watermarking in transform domain is to give more robustness in the watermarking process looking into its fast real time implementation and also the computational complexity which comes often in the implementation process. In the proposed algorithm, a binary watermark is embedded in a grayscale image(cover image). The cover image is segregated into blocks of high and low variances. The cover image is transformed into frequency domain and comparative higher variance blocks are considered for embedding the binary watermark. Integer portion of the transformed DC coefficients of the considered blocks undergoes binary conversion and binary bits of watermark are embedded in those co-efficients. Both Walsh Transform and Walsh Hadamard transform are used for frequency domain conversion of the image and to make a comparative study as well. Software simulation of the algorithm is done using MATLAB
\end{abstract}

\section{Keywords}

VARIANCE, WALSH, HADAMARD, VERILOG

\section{INTRODUCTION}

In the field of digital media, the safety and security of the digital information is always a matter of concern since the digital information can easily be manipulated with the help of computers and other digital devices. So, gradually the needs of some techniques were felt to protect the identity and ownership of digital information and to authenticate those digital content. One such technique is Digital Watermarking. In digital Image watermarking, any digital information (message data) which is generally termed as 'Watermark' is embedded into a carrier image without causing permanent damage to the carrier image and the watermark as well, so that the information remains protected in its original form. For hiding the message, the encryption or watermark insertion algorithm should be robust and also it should be immune against various noises and attacks during the transmission or sharing process. In this proposed work, watermarking is performed in transform domain and then the watermark is inserted and embedded with the transformed values according to the algorithm in a robust manner. In the proposed work, Walsh transform \& Hadamard transform is used to establish the transform domain.

\section{RELATED WORKS}

Many researchers have been working in this domain of digital watermarking. I. J. Cox at el presented a secure (tamperresistant) algorithm for watermarking images, in the proposed methodology a watermark should be constructed as an independent and identically distributed Gaussian random vector that is imperceptibly inserted in a spread-spectrum-like fashion into the perceptually most significant spectral components of the data[1]. Hesham A. El Zouka proposed a new approach where a key based on a seed number is used to communicate and generate a sequence of numbers that identifies bits in the host digital media file that contains the secret message [2]. Gogoi et al proposed an algorithm which can embed double watermarks in a single cover image, it was further extended to video as well [3]. A low power, real time, reliable and secure data hiding system that can be achieved all the way through hardware implementation was proposed by Abhishek Basu at el[4]. A real time implementation of an algorithm for Watermarking and the results obtained using a Frequency approach by modifying the middle frequency coefficients was put forward by Ravi Shah [6]. Kishan Chand Gupta and Palash Sarkar studied the relationship between the Walsh transform and the algebraic normal form (ANF) of a Boolean function. They proposed an algorithm which can be applied in situations where it is practically impossible to use the fast Walsh transform algorithm [7]. A transform domain watermarking implementation was carried out by S. Yan at el they proposed a novel combined multi-polarity arithmeticWalsh transform, in which recursive relationships between higher and lower matrix orders of hybrid multi-polarity arithmetic-Walsh transform are developed [8]. Fu Jun and Wang Shou-huai presented a Walsh code orthogonal spread spectrum watermarking algorithm in which the watermark is embedded into the medium frequency and low frequency of the carrier block, according to the visual masking effect of the human visual system[9]. D.Sasikala et al proposed a registration method on medical images using Fast Walsh Hadamard Transform, each basis function of Walsh Transform is a notion for determining various aspects of local structure. The co-efficients are normalized and used in forming a unique number for each type of local structure [10]. Patrick Gaydecki et al proposed a technique which inserts the binary bits of the handwritten signatures in the DCT blocks of $\mathrm{Y}$ channel of the digital colour image as well as uses 1-D walsh coding to embed mobile phone digits in images captured by the mobile[11]. Koushik Mahanta et al proposed a hardware watermarking technique where watermark embedding is done using a MSI number in walsh domain[12]. 


\section{MODELLING OF WATERMARKING SCHEME}

stages of the flow of computing and are shown in the Fig1 and Fig 2.

The transmitter and receiver block contains several components which are used at various

Transmitter Block:

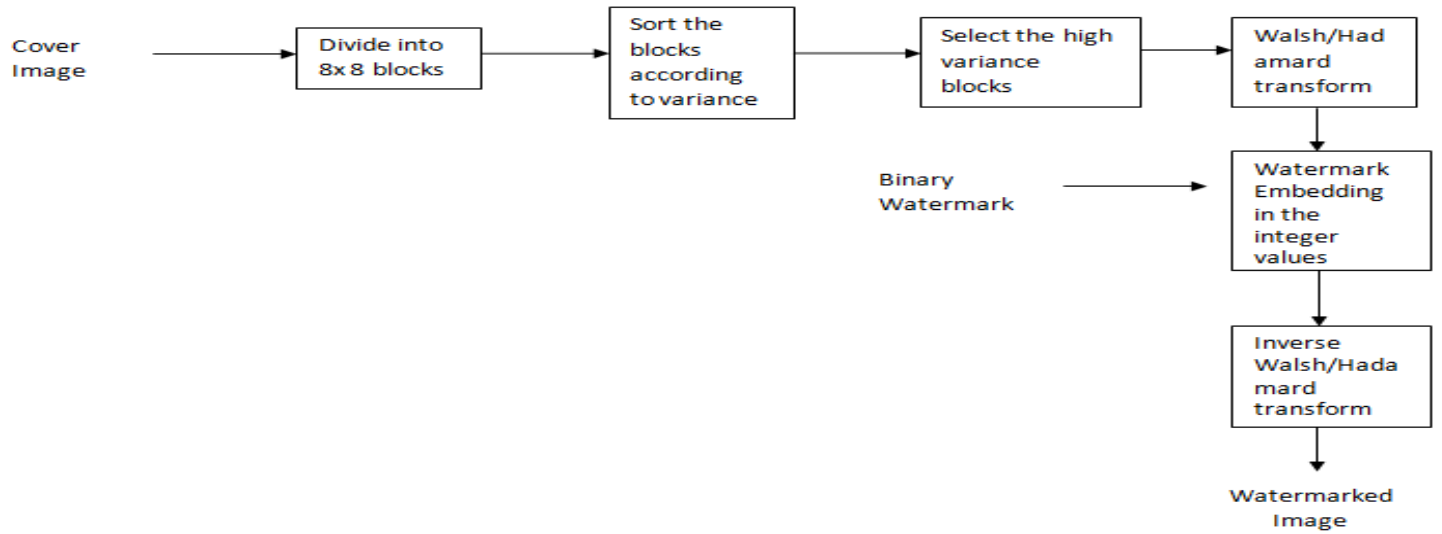

Fig1. Block Diagram of Transmitter

Receiver Block:

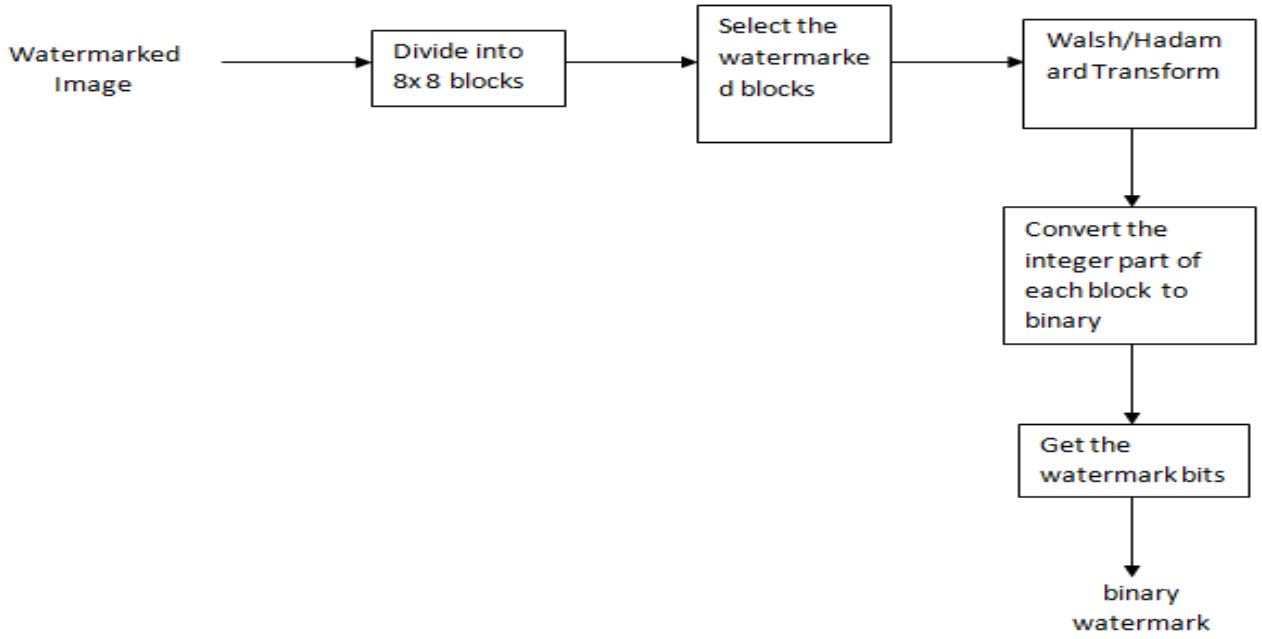

Fig2. Block Diagram of Receiver

\section{THEORETICAL DETAILS OF THE TRANSFORM FUNCTIONS:}

\subsection{Theoretical details of Walsh Transform}

The kernel of the Fast Walsh Transform can be given as:

$$
\mathrm{f}(\mathrm{x}, \mathrm{u})=\pi_{i=0}^{k-1}\left((-1)^{b_{i}(x) b_{k-1-i}(u)}\right) * \frac{1}{N}
$$

where $\mathrm{N}=$ No. of samples from an image

$\mathrm{k}=$ number of bits needed to represent $\mathrm{x}$ as well as $\mathrm{u}$ such that $\mathrm{N}=2^{\mathrm{k}}$.

$b_{i}(x)$ means $i^{\text {th }}$ bit of the binary representation of $x$. Similar is the case with $b_{\mathrm{k}-1-\mathrm{i}}(\mathrm{u})$.

$\mathrm{W}(\mathrm{u})=\left(\sum_{x=0}^{n-1} g(x)\left[\left(\pi_{i=0}^{n-1}\left((-1)^{b_{i}(x) b_{n-1-i}(u)}\right)\right) * \frac{1}{N}\right]\right.$

where $\mathrm{g}(\mathrm{x})$ is the input image pixel.
The inverse kernel of the Fast Walsh Transform is same as the forward kernel of Fast Walsh Transform i.e.

$$
\mathrm{f}^{\prime}(\mathrm{x}, \mathrm{u})=\pi_{i=0}^{n-1}\left((-1)^{b_{i}(x) b_{n-1-i}(u)}\right) * \frac{1}{N}
$$

Inversed transform:

$$
\operatorname{IW}(\mathrm{u})=\mathrm{g}^{\prime}(\mathrm{x}) \times \mathrm{N} \times \mathrm{f}^{\prime}(\mathrm{x}, \mathrm{u})
$$

i.e $\operatorname{IW}(\mathrm{u})=\sum_{x=0}^{n-1} g^{\prime}(x)\left[\left(\pi_{i=0}^{n-1}\left((-1)^{b_{i}(x) b_{n-1-i}(u)}\right)\right)\right.$

Where $\mathrm{g}^{\prime}(\mathrm{x})=\mathrm{W}(\mathrm{u})$;

\subsection{Theoretical details of the Hadamard Transform}

Kernel of Walsh Hadamard Transform can be given as-

$$
\mathrm{h}(\mathrm{x}, \mathrm{u})=\left((-1)^{\sum_{i=0}^{n-1} b_{i}(x) b_{i}(u)}\right) * \frac{1}{N}
$$


where $\mathrm{N}=$ No. of samples from an image

$\mathrm{k}^{\prime}=$ number of bits needed to represent $\mathrm{x}$ as well as $\mathrm{u}$ such that $\mathrm{N}=2^{\mathrm{k}^{\prime}} . \mathrm{b}_{\mathrm{i}}(\mathrm{x})$ means $\mathrm{i}^{\text {th }}$ bit of the binary representation of $\mathrm{x}$. Similar is the case with $\mathrm{b}_{\mathrm{i}}(\mathrm{u})$.

$$
\mathrm{W}_{\mathrm{H}}(\mathrm{u})=\sum_{x=0}^{n-1} f(x)\left[\left((-1)^{\sum_{i=0}^{n-1} b_{i}(x) b_{i}(u)}\right) * \frac{1}{N}\right]
$$

$\mathrm{f}(\mathrm{x})=$ Original Image

The inverse kernel of the Hadamard Transform is same as the forward kernel of Hadamard Transform i.e.

$$
\mathrm{h}^{\prime}(\mathrm{x}, \mathrm{u})=\left((-1)^{\sum_{i=0}^{n-1} b_{i}(x) b_{i}(\mathrm{u})}\right) * \frac{1}{N}
$$

Inverse transform is given by:

$\mathrm{IW}_{\mathrm{H}}(\mathrm{u})=\sum_{x=0}^{n-1} m^{\prime}(x)\left[\left((-1)^{\sum_{i=0}^{n-1} b_{i}(x) b_{i}(u)}\right)\right]$

Where $\mathrm{m}^{\prime}(\mathrm{u})=\mathrm{W}_{\mathrm{H}}(\mathrm{u})$

\section{PROPOSED WATERMARKING ALGORITHM}

There are two parts in the algorithm. One part is for the watermark encoding process and the other part is for the watermark decoding process

\subsection{Watermark encoding}

Step 1: Take the cover image and divide it into (8x8) no of blocks.

Step 2: Sort the blocks according to the variance in descending order.

Step 3: Select the higher variance blocks.

Step 4: Perform walsh/Hadamard transform to all the blocks.

Step 5: Take the watermark image which is of 256 bits (16 $x$ 16 binary image).

Step 6: Watermark Embedding

(i)Two watermark bits are added to each $8 \times 8$ block

(ii) Each $8 \times 8$ block contribute 8 DC components.

(iii) The integer part of each DC component is converted to binary with 8 bits.

(iv)The LSB $0^{\text {th }}$ and $1^{\text {st }}$ positions / LSB $1^{\text {st }}$ and $2^{\text {nd }}$ positions of each DC component are replaced by the same two bits.

\subsection{Watermark decoding}

Step 1: Take the watermarked Image and divide it into (8x8) number of blocks.

Step 2: Perform walsh/hadamard transform to all the blocks.

Step3:Select the watermark embedded blocks.

Step 4 : For each block binary form.

(i) Select the eight DC components and convert to

(ii) Get the LSB $0^{\text {th }}$ and $1^{\text {st }}$ positions / LSB $1^{\text {st }}$ and $2^{\text {nd }}$ positions of each component and count no. of 1 's for both. In each position consider the following

$$
\begin{aligned}
& \text { If (no. of } 1 \text { 's }>\text { no. of } 0 \text { 's) } \\
& \text { extracted bit }=1 \\
& \text { else } \\
& \text { extracted bit }=0
\end{aligned}
$$

\section{MATLAB SIMULATION}

By following the Watermarking algorithm a detailed comparison of the watermarked image has been performed for the two domains (i.e-Walsh Transform and Hadamard Transform). The original image, watermarked image and the watermark locations have been shown in Fig3, Fig4 and Fig5.

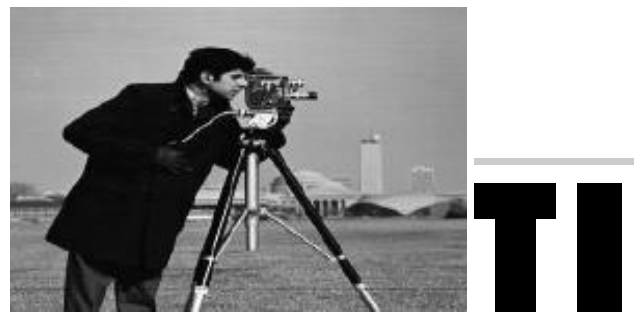

Fig3-Cover Image

Fig 4-Watermark

The PSNR value of the watermarked image with watermarked embedded at different positions for Walsh Transform and Walsh hadamard Transform shown in TABLE I \& II

Walsh Transform:

For bit positions $\left(1^{\text {st }} \& 2^{\text {nd }}\right), P S N R=48.34$ and for bits positions $\left(1^{\text {st }} \& 0^{\text {th }}\right)$, PSNR $=54.28$

Step 7: Perform Inverse Walsh Transform

Table I: Extracted watermarks and PSNR values.

\begin{tabular}{|c|c|c|l|l|}
\hline $\begin{array}{l}\text { Watermarkin } \\
\text { g } \\
\text { Attacks }\end{array}$ & \multicolumn{3}{|c|}{ Watermark image } & $\begin{array}{l}\text { PSNR values } \\
\text { (Walsh Transform })\end{array}$ \\
\hline & $\begin{array}{c}\text { bits positions }\left(1^{\text {st }}\right. \\
\left.\& 2^{\text {nd }}\right)\end{array}$ & $\begin{array}{c}\text { bits positions }\left(1^{\text {st }} \&\right. \\
\left.0^{\text {th }}\right)\end{array}$ & \multicolumn{2}{c|}{ Watermarks } \\
\hline $\begin{array}{c}\text { Gaussian } \\
\text { Filter }\end{array}$ & & \\
\end{tabular}




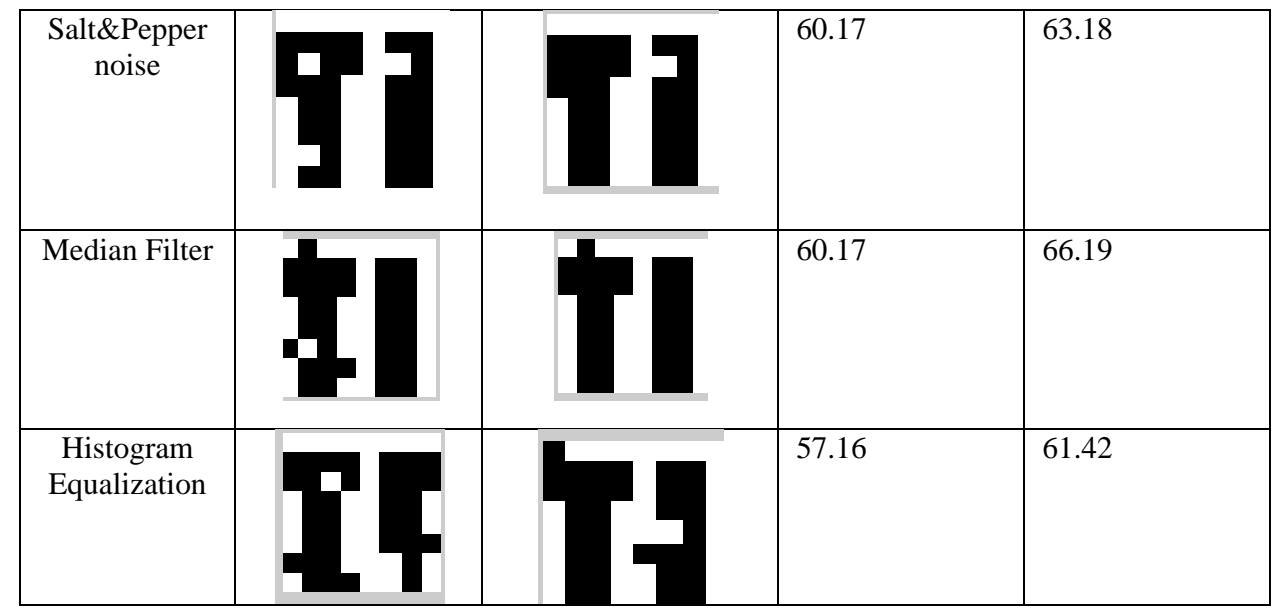

Walsh Hadamard Transform:

For bit positions $\left(1^{\text {st }} \& 2^{\text {nd }}\right), P S N R=56.19$ and for bits positions $\left(1^{\text {st }} \& 0^{\text {th }}\right)$, PSNR $=50.2$

Table II: Extracted watermarks and PSNR values

\begin{tabular}{|c|c|c|c|c|}
\hline $\begin{array}{l}\text { Watermarkin } \\
\mathrm{g}\end{array}$ & \multicolumn{2}{|c|}{ Watermark image } & \multicolumn{2}{|c|}{$\begin{array}{l}\text { PSNR values } \\
\text { (Walsh Hadamard Transform) }\end{array}$} \\
\hline & bits positions $\left(1^{\text {st }}\right.$ & bits positions $\left(1^{\text {st }} \&\right.$ & \multicolumn{2}{|c|}{ Watermarks } \\
\hline $\begin{array}{l}\text { Gaussian } \\
\text { Filter }\end{array}$ & & & $\begin{array}{l}\text { bits positions }\left(1^{\text {st }}\right. \\
\left.\qquad \& 2^{\text {nd }}\right)\end{array}$ & $\begin{array}{l}\text { bits positions }\left(1^{\text {st }}\right. \\
\left.\& 0^{\text {th }}\right)\end{array}$ \\
\hline & & & 52.21 & 57.74 \\
\hline $\begin{array}{l}\text { Salt\&Pepper } \\
\text { noise }\end{array}$ & & & 58.41 & 66.12 \\
\hline Median Filter & & & 63.18 & 66.19 \\
\hline $\begin{array}{l}\text { Histogram } \\
\text { Equalization }\end{array}$ & & & -------- & --------- \\
\hline
\end{tabular}

The experimental result shows that the PSNR values of the extracted watermarks are very much acceptable for both the transform. The algorithm was implemented in 50 gray scales images all showed satisfactory results.

\section{CONCLUSION}

The design of Digital Image Watermarking in transform domain is done using Walsh and Hadamard Transform mainly for their robustness against different image processing attacks. The designing process will reduce the complexity of the circuit when extended to hardware design as the transform domain does not involve any imaginary co-efficient. The algorithm can also be implemented for video watermarking. Apart from the attacks mentioned above there is also a future scope of extending the watermarking algorithm to increase its robustness for more attacks like rotating etc.

\section{REFERENCES}

[1] Ingemar J. Cox, Joe Kilian, Tom Leighton and Talal Shamoon, Secure Spread Spectrum Watermarking for Multimedia, IEEE Trans. on Image Processing, 6, 12, 1673-1687, (1997). Page 1-31

[2] Hesham A. El Zouka, Computer Engineering Department, Arab Academy for Science and Technology, Alexandria - Egypt, FPGA Based Implementation of Robust Watermarking System, Fifth International Conference on Information Technology: New Generations, Pages 1-5 
[3] Mriganka Gogoi, H.M.Khalid Raihan Bhuyan, Koushik Mahanta, Dibya Jyoti Das, Ankita Dutta Image and Video based double watermark extraction spread spectrum watermarking in low variance region, International Journal of Advanced Computer Science and Applications Vol.4, No. 6, 2013

[4] Abhishek Basu, Tirtha Sankar Das, Somnath Maiti, Nurul Islam and Subir Kumar Sarkar, FPGA Based Implementation of Robust Spatial Domain Image Watermarking Algorithm, International Conference on Computers and Devices for Communication,2009, Page $1-4$

[5] Amit M Joshi, Anand Darji and Vivekanand Mishra, Electronics Department, SVNIT, Surat, India, Design and Implementation of Real-Time Image Watermarking, Page 1-5

[6] Ravi Shah, Abhinav Agarwal and Subramaniam Ganesan, Department of Computer Science and Engineering, Oakland University, MI-48309, Frequency Domain Real Time Digital Image Watermarking, Page 1-6

[7] Kishan Chand Gupta and Palash Sarkar, Computing Partial Walsh Transform From the Algebraic Normal Form of a Boolean Function, IEEE Transactions on Information Theory, Vol. 55, No. 3, March 2009, Pages $1-6$

[8] B.J. Falkowski and S. Yan, Hybrid multi-polarity arithmetic-Walsh transform, IEEE Proc.-Circuits Devices Syst., Vol. 153, No. 6, December 2006, Pages 18

[9] Fu Jun and Wang Shou-huai, An adaptive spread spectrum watermarking algorithm based on Orthogonal Walsh codes, Pages 1-3

[9] Patrick Gaydecki, Kamal A. Ahmed and Hussain Al Ahmad, A Blind Block Based DCT Watermarking Technique for Gray Level Images Using One Dimensional Walsh Coding, Pages 1-5
[10] D.Sasikala and R.Neelaveni, Correlation Coefficient For Registration Of Monomodal Brain Images Using Fast Walsh Hadamard Transform, ICCCCT'10, Pages 1-6

[11] Patrick Gaydecki, Kamal A. Ahmed and Hussain Al Ahmad, A Blind Block Based DCT Watermarking Technique for Gray Level Images Using One Dimensional Walsh Coding, Pages 1-5

[12] Koushik Mahanta, Dibya Jyoti Das, H. M. Khalid Raihan Bhuyan, Ankita Dutta, Mriganka Gogoi, Design and implementation of an MSI number based image watermarking architecture in transform domain, 2014 International Conference on Signal Processing and Integrated Networks (SPIN). 2014; p. 157-63.

[13] Fu Jun and Wang Shou-huai, An adaptive spread spectrum watermarking algorithm based on Orthogonal Walsh codes, Pages 1-3

[14] Zulfikar, Shuja A. Abbasi and A. R. M. Alamoud, A Novel Complete Set of Walsh and Inverse Walsh Transforms for Signal Processing, International Conference on Communication Systems and Network Technologies 2011, Pages 1-6

[15] Kamal A. Ahmed, Hussain Al-Ahmad and Patrick Gaydecki, Robust Image Watermarking Using Two Dimensional Walsh Coding, Pages 1-5

[16] Sivavenkateswara Rao . V., Rajendra S. Shekhawat and V. K. Srivastava, A DWT-DCT-SVD Based Digital Image Watermarking Scheme Using Particle Swarm Optimization, IEEE Students' Conference on Electrical, Electronics and Computer Science 2012, Pages 1-4

[17] Zhe-Ming Lu, Hans Burkhardt and Shu-Chuan Chu, Multipurpose Image Watermarking Algorithms and Applications, Studies in Computational Intelligence (SCI) 58, 287-323 (2007),Pages 287-288 